Res Pública Revista de Historia de las Ideas Políticas

ISSN: $1131-558 \mathrm{X}$

\title{
Escribiendo sobre Weimar y el (neo)nazismo desde México: Paul Merker y Vicente Lombardo Toledano
}

\author{
Celia Alejandra Ramírez Santos*
}

Recibido: 8 de febrero de 2019 / Aceptado: 16 de octubre de 2019

\begin{abstract}
Resumen. En 1944 se editó en México La caída de la República de Weimar, traducción al castellano de la primera parte del libro Deutschland, Sein oder nicht Sein, escrita por el comunista alemán exiliado en México, Paul Merker. El libro cuenta con dos prólogos significativos, uno a cargo del mismo Merker y otro escrito por Vicente Lombardo Toledano, figura central del socialismo mexicano en el siglo XX. Estos prólogos resultan interesantes para entender las distintas vías al socialismo ensayadas por entonces en Europa y en América Latina. El libro de Merker constituye, además, una historia crítica de la República de Weimar como un "laboratorio" de ensayo y preparación de estrategias autoritarias y violentas contra el bolchevismo, en oposición a la visión idealizada - hoy en día predominante- de la República de Weimar como la primera democracia alemana y una página gloriosa de su socialdemocracia.
\end{abstract}

Palabras clave: República de Weimar; socialdemocracia; comunismo; laboratorio; nazismo; exilio alemán en México.

[en] Writing about Weimar and (Neo)Nazism from Mexico: Paul Merker and Vicente Lombardo Toledano

Abstract. In 1944 appeared in Mexico La caída de la República de Weimar, translation into Castilian of the first part of the book Deutschland, Sein oder nicht Sein, written by Paul Merker, German communist exiled in Mexico. The book includes two interesting prefaces written by Merker and by Vicente Lombardo Toledano, a key figure of Mexican socialism in the twentieth century. Those introductory texts are interesting in order to understand the different ways into socialism that, by the middle of the 1940s, were put into practice in Europe and Latin America. Merker's book also represents a critical history of the Weimar Republic, conceived as a "laboratory" of authoritarian and violent strategies against bolshevism. His vision is at odds with the idealized perspective -today predominant- of the Weimar Republic as the first German democracy and a glorious episode in the history of its social democracy.

Keywords: Weimar Republic; Social democracy; Communism; Laboratory; Nazism; German exile in Mexico.

Sumario. 1. La caída de la República Alemana de Paul Merker, Lombardo Toledano y el exilio alemán en México. 2. Principales críticas de Merker a la República de Weimar: nacionalismo imperialista, militarismo y socialdemocracia en "el laboratorio de los enemigos del progreso". 3. El prólogo exculpatorio "al lector latinoamericano". 4. Afinidades y diferencias entre la república revolucionaria mexicana y la democracia imperialista de Weimar. 5. Conclusiones. Lombardo Toledano y el (neo)nazismo, una relación duradera. 6 . Bibliografía.

Cómo citar: Ramírez Santos, C. A. (2020). Escribiendo sobre Weimar y el (neo)nazismo desde México: Paul Merker y Vicente Lombardo Toledano. Res Pública. Revista de Historia de las Ideas Políticas, 23(1), 37-45.

\section{La caída de la República Alemana de Paul Merker, Lombardo Toledano y el exilio alemán en México}

En 1944 se editó en la Ciudad de México la obra de Paul Merker titulada La caída de la República Alemana, el ca- mino de Hitler al poder ${ }^{2}$. Publicada por la editorial El Libro Libre, que aglutinó a los intelectuales comunistas alemanes exiliados en México desde su fundación en 1942 y hasta el final de la Segunda Guerra Mundial en $1945^{3}$, la obra es la traducción al castellano -realizada por el exiliado espa-

Universidad Complutense de Madrid

alejandra.ramirez.filo@gmail.com

P. Merker, La caída de la República Alemana. El camino de Hitler al poder, México, El Libro Libre, 1944.

2 Sobre los exiliados comunistas alemanes en México y la editorial El Libro Libre, cf.: W. Kießling, Exil in Lateinamerika. Kunst und Literatur im antifaschistischen Exil 1933-1944, Leipzig, Reclam, 1980; Viva Mexico. Brücken nach Mexiko. - Tradition einer Freundschaft, Dietz Verlag, Berlín, 1989; Alemania Libre in Mexiko. Band 1. Ein Beitrag Zur Geschichte Des Antifaschistischen Exils 1941 - 1946, Band 2. Texte Und Dokumente Zur Geschichte Des Antifaschistischen Exils 1941 - 1946, Berlín, Akademie Verlag, 1974. 
ñol Manuel Andújar ${ }^{3}$ - de la primera parte del libro de Paul Merker, Deutschland. Sein oder nicht Sein?4.

Un par de prólogos, escritos por el propio Paul Merker y por Vicente Lombardo Toledano, quien había sido Secretario General de la Confederación de Trabajadores de México (CTM), el gran sindicato mexicano, durante el gobierno de Lázaro Cárdenas (1934-40) y se encargó de recibir y emplear a los exiliados europeos durante la Segunda Guerra Mundial ${ }^{5}$, distingue a la edición de la obra en castellano del trabajo original en alemán.

La introducción de Lombardo Toledano es, en este caso, menos significativa que otros trabajos suyos sobre el nazismo escritos antes y después de $1944^{6}$. En esta introducción, particularmente, caracteriza al libro de Merker como imprescindible para la comprensión de lo que había sucedido en Alemania a partir de 1918 y le da valor como "libro antinazi" y "libro de combate" escrito por alguien que "durante años ha sido un destacado dirigente de las fuerzas subterráneas -hasta ahora sólo en minoría- que dentro del Tercer Reich libran la batalla contra la barbarie"7.

En efecto, sin llegar a ser uno de los dirigentes más destacados del Partido Comunista Alemán (KPD), Merker contaba con una larga trayectoria en el mismo. Con una cierta afinidad con la carrera política y sindical del propio Lombardo Toledano, Merker había trabajado en la década de los veinte en el departamento del Partido Comunista Alemán en-

Su figura conoce un creciente interés por parte de la historiografía filosófica y literaria: J.L. Abellán, "Manuel Andújar, literatura y conciencia", Cuadernos Hispanoamericanos 529-530, 1994, pp. 281292; G. Hervás Fernández, "Manuel Andújar, un escritor del exilio", Revista de Filología Románica 28, 2011, pp. 163-170. En 2011 le fue dedicado un número especial del Boletín del Instituto de Estudios Giennenses con contribuciones de varios autores. Destaca el trabajo de R. De Cózar, "Manuel Andújar y el exilio", Boletín del Instituto de Estudios Giennenses 211, 2015, pp. 281-292; V. Vegazo Palacios, "Manuel Andújar, el exilio y la vindicación de la memoria histórica", Campo de Agramante: revista de literatura 9, 2008, pp. 95-108.

4 Se trata de uno de los seis libros que la editorial publicó en castellano. El resto de títulos, veinte, fueron publicados en alemán. La caída de la República Alemana había sido editada también en alemán ese mismo año en El libro libre con el título escueto Von Weimar zu Hitler. P. Merker, Deutschland - Sein oder Nicht Sein?. Band 1. Von Weimar zu Hitler, México, El Libro Libre, 1944; Band 2, Das Dritte Reich und sein Ende, México, El Libro Libre, 1945.

5 Lombardo Toledano impulsó con gran ahínco las asociaciones antifascistas Liga Pro Cultura Alemana y Alemania Libre (a partir de 1942). Un artículo publicado recientemente por Andrea Acle-Kreysing en la Revista de Indias se concentra en estudiar el apoyo ofrecido por Lombardo a los exilios alemán y español en México. El artículo resulta especialmente interesante por su análisis de las afinidades y divergencias ideológicas entre los antifascistas mexicanos y europeos. A. Acle-Kreysing, "Antifascismo: un espacio de encuentro entre el exilio y la política nacional. El caso de Vicente Lombardo Toledano en México (1936-1945)", Revista de Indias LXXVI-267, 2016, pp. 573-609.

6 V. Lombardo Toledano, ¿Cómo actúan los Nazis en México?, México, Universidad Obrera de México, 1941; Una intriga nazi contra la defensa del Continente Americano, Universidad Obrera de México, México, 1942; Judios y mexicanos: ¿razas inferiores?, Universidad Obrera de México, 1942. Destaca también un pequeño tratado que publicó dos décadas después por su empleo pionero del concepto neonazismo, El neonazismo: sus características y peligros, México, Escuela Nacional de Ciencias Políticas y Sociales, 1960

V. Lombardo Toledano, "Introducción", en P. Merker, La caída de la República Alemana. El camino de Hitler al poder, México, El Libro Libre, 1944, p. 13. cargado de hacer valer las líneas de acción del partido en la esfera sindical. A principios de los treinta se desempeñó como agente al servicio de la Internacional Comunista en Estados Unidos y la Unión Soviética. Desde la llegada al poder del nazismo y la prohibición del Partido Comunista Alemán, Merker siguió luchando en la clandestinidad, tanto dentro como fuera de Alemania. En esta época fue miembro de los principales órganos directivos del Partido. Tras un primer largo exilio en Francia, la presión de la GESTAPO lo lleva a abandonar Europa en 1942 y a encontrar refugio junto a otros comunistas alemanes en México. En el México post-cardenista, presidido entonces por Manuel Ávila Camacho (1940-46), la figura de Merker emergió como la del "líder indiscutible del pequeño grupo del KPD en México"8, desempeñando simultáneamente los cargos de secretario del Comité Latinoamericano por una Alemania libre, editor de la revista Freies Deutschland e impulsor, junto a Lombardo Toledano, de la editorial El Libro Libre.

Aunque el dato apuntado por Lombardo al afirmar que "éste es el primer libro sobre Alemania escrito por un hombre que ha trabajado en la clandestinidad del imperio hitleriano" no es del todo cierto, sí es verdad que se trata de una fuente coetánea de gran importancia. Dado el anti-comunismo imperante en la Guerra Fría, versiones de lo acontecido durante la República de Weimar como la del socialdemócrata Arthur Rosenberg, quien ya en 1935 había publicado un libro sobre el tema, han gozado, sin embargo, de mayor atención editorial y eco mediático hasta fechas recientes ${ }^{9}$. La poca atención dedicada a Merker y su obra se extiende en realidad a la práctica totalidad de los miembros del circulo antifacista fundado por la escritora Anna Seghers en Ciudad de México. Personajes incomodos para el régimen de Weimar y el nazismo, lo siguen siendo para la Alemania actual, que parece resistirse a darles $\mathrm{voz}^{10}$. Con excepción de unos pocos libros y artículos de revista se ha trabajado poco sobre ellos y sobre su

\footnotetext{
Un sucinto relato sobre sus actividades en México en C. Epstein, The Last Revolutionaries: German communists and their century, Cambridge, Harvard University Press, 2003, pp. 9-91. La literatura sobre Merker ha experimentado un notable crecimiento en las últimas décadas. Presentan secciones amplias sobre las actividades de Merker en México los trabajos ya mencionados de Kießling y otros que el gran historiador de la Alemania Democrática dedicó específicamente a la figura de Merker: W. Kießling, Paul Merker in den Fängen der Sicherheitsorgane Stalins und Ulbrichts, Berlín, Gesellschaftswissenschaftliches Forum, 1995; Partner im Narrenparadies. Der Freundeskreis um Noel Field und Paul Merker, Dietz Verlag, Berlin, 1994.

$9 \quad$ La historia escrita por Arthur Rosenberg, miembro en la década de los veinte del KPD y, más tarde, crítico socialdemócrata del mismo, fue publicada en la temprana fecha de 1935 y goza de un mayor reconocimiento por los aparatos académicos contemporáneos, habiendo sido reeditada en decenas de ocasiones. A. Rosenberg, Entstehung und Geschichte der Weimarer Republik, Karslbad, Graphia, 1935.

10 Lo que contrasta con su gran reconocimiento internacional. Una biografía reciente de Marike Janzen se concentra en la vida de Seghers, concediendo gran importancia a su exilio en México, M. Janzen, Writing to Change the World: Anna Seghers, Authorship, and International Solidarity in the Twentieth Century, Rochester, Candem House, 2018.
} 
producción escrita e iniciativas editoriales y periodísticas en México ${ }^{11}$.

Pese a su escasa consideración en la misma Alemania, La caída de la República Alemana de Paul Merker nos transmite una perspectiva sobre el régimen de Weimar que, aunque marcadamente comunista, no cae en maniqueísmos. Su misma edición en México, por entonces mucho más cercana a la socialdemocracia que al comunismo, puede ser considerada un indicio del valor de una obra que, como afirma Lombardo en la "Introducción" mencionada, "no excluye al Partido Comunista Alemán de las críticas que dirige a los demás partidos y agrupaciones antinazis" y "no trata de atenuar la gran responsabilidad del pueblo alemán y de la clase obrera alemana"12 en el triunfo electoral del nazismo y su consolidación al frente del Estado alemán en el período 1933-45.

\section{Principales críticas de Merker a la República de Weimar: nacionalismo imperialista, militarismo y socialdemocracia en "el laboratorio de los enemigos del progreso"}

De acuerdo a la interpretación de Merker, la principal causa de la caída de la República de Weimar había sido su ausencia de una ruptura clara y decidida con el militarismo e imperialismo del Segundo Reich Alemán y el trabajo conjunto de los partidos burgueses, incluido el Partido Socialista Alemán (SPD), con el ejército y tropas paraestatales organizadas para reprimir a los movimientos obreros.

Inseguros de sus propias fuerzas y creyendo quizás que los militares serían el brazo protector de la democracia de Weimar frente a los movimientos revolucionarios tendentes a la instauración de una dictadura del proletariado, liberales y socialdemócratas aceptaron colaborar estrechamente con la misma élite militar y nobiliaria que había llevado a Alemania a la derrota de 1918.

Tal colaboración no se produjo, de acuerdo a Merker, de una forma accidental ni ocasional. Tampoco fue una especie de último recurso de los demócratas burgueses en un contexto de excepción. En su opinión, la República de Weimar había nacido ya, en noviembre de 1918, con un carácter antidemocrático y con el objetivo esencial de impedir el acceso de los comunistas - masivamente apoyados por el proletariado alemán- al poder.

En la "alianza concertada" secretamente "el 10 de noviembre de 1918, un día después de la proclamación de la República alemana, por el primer Presidente [de la República, el SPDista Friedrich Ebert], con el último

11 Destacan los trabajos de F. Pohle, Das Mexicanische Exil: ein Beitrag zur Geschichte der politisch-kulturellen Emigration aus Deutschland (1937-1946), Stuttgart, Metzler, 1986; R. Hanffstengl, Mexiko, das wohltemperierte Exil, México, Instituto de Investigaciones Interculturales Germano-Mexicanas, 1995; F. Katz, "El exilio centroeuropeo. Una mirada autobiográfica”, en P. Yankelevich, México, país refugio: la experiencia de los exilios en el siglo XX, México, CONACULTA / INAH, 2002, pp. 43-48; R. Pérez Montfort, “Apuntes sobre el exilio alemán en México", en ibidem, pp. 49-54; D. Gleizer, Unwelcome exiles: Mexico and the Jewish refugees from Nazism, 1933-1945, Leiden, Brill, 2013; T. Cañadas García, La huella de la cultura en lengua alemana en México a partir del exilio de 1939-1945, Madrid, Fundación Universitaria Española, 2016.

12 V. Lombardo Toledano, "Introducción“, op. cit., p. 14. mariscal del Káiser Guillermo II, Paul von Hindenburg" y su adjunto Wilhelm Groener ubica Merkel el acto de fundación de una república alemana concebida como un instrumento para frenar por todos los medios a su alcance el "peligro bolchevique"13.

En la disyuntiva coyuntural de apoyar al ejército profesional de la élite imperialista o a los Consejos de Obreros Soldados que, en estas fechas, y tal como había sucedido un año antes en Rusia, estaban surgiendo por toda Alemania para llevar a cabo una revolución que acabara con el Segundo Reich ${ }^{14}$, los socialdemócratas optaron por aliarse con las élites tradicionales.

De acuerdo con Merker, el pacto Ebert-Groener selló también el miserable destino de la República de Weimar. Salvando a los militares del Reich en su momento más crítico, quizás con la esperanza de que se convirtieran en guardianes de la nueva democracia, los socialdemócratas cometieron un error fatal. Sin ningún propósito de enmienda ni ningún compromiso con los valores democráticos, los militares y las viejas élites tomaron simplemente la República de Weimar como un paréntesis de recuperación de fuerzas y preparación para una nueva guerra.

En este sentido, dice Merker en su "Prólogo al lector latinoamericano": "Ciertamente que la democracia de Weimar no se hundió por el supuesto concepto de que los alemanes no pudieran ser republicanos ni demócratas. Feneció porque los gobiernos llamados a guardar la Constitución democrática y ampliarla, se colocaron al servicio del imperialismo germano"15.

La perspectiva de Merker está, claramente, en el extremo opuesto de la visión de la historia que, andando el tiempo, se convertirá en la línea oficial de interpretación de la República de Weimar en la República Federal Alemana, a saber, la que hace al SPD de Friedrich Ebert y los partidos liberales y conservadores que, junto al SPD integraron la Weimarer Koalition, los únicos y auténticos defensores de la democracia en un contexto político marcado por el peso cada vez más creciente de los extremistas anti-democráticos de derecha (nacionalsocialismo) e izquierda ${ }^{16}$.

\footnotetext{
Fueran legales y parlamentarios, o ejecuciones extrajudiciales a cargo de varios cuerpos de asesinos y espías entre los que figuraba el mismo Hitler.

14 El SPD no sólo apostó por gobernar la República en coalición con distintos partidos conservadores alemanes, sino que incluso apoyó a los primeros cuerpos paramilitares encargados de reprimir al movimiento comunista desde la frustrada y efímera República Socialista de Baviera (1918-19).

15 P. Merker, "Prólogo al lector latinoamericano", en P. Merker, La caída de la República Alemana. El camino de Hitler al poder, op. cit., p. 15. Su perspectiva es radicalmente inversa a la adoptada por la historiografía

16 Tal postura historiográfica se construye ya en obras de finales de los años 40, destinadas a "blanquear" la responsabilidad de los socialdemócratas y sus aliados centristas - es decir, las mismas fuerzas que monopolizarán el poder en la RFA después de la Segunda Guerra Mundial- en la creación de los cuerpos paramilitares y parapoliciales de los que Hitler formó parte y que, más tarde, utilizó para acceder al poder. O. Flechtheim, Der KPD in der Weimarer Republik, Offenbach, Bollwerk/Karl Drott, 1948. A. Wirsching, Vom Weltkrieg zum Bürgerkrieg? Politischer Extremismus in Deutschland und Frankreich 1918 - 1933/9, Munich, Oldenbourg, 1999. Más afín a la perspectiva que Merker ofrece de forma pionera son los trabajos de la última generación de historiadores formados en la República Democrática Alemana. Cf., por ejemplo, K. Kinner, Der deutsche Kommunismus 1. Die Weimarer Zeit: Selbstverständnis und Realität, Berlín, Dietz, 1999.
} 
Resulta muy interesante que, en el marco de sus críticas a la República de Weimar, Paul Merker emplee el calificativo de "laboratorio" para referirse a las distintas estrategias empleadas por los grandes conglomerados industriales y las fuerzas reaccionarias para intentar frenar los avances de un movimiento obrero cuyo triunfo parecía inminente tras la llegada al poder de los soviets. Ello prueba que no se trata de un planteamiento artificial ni de un calificativo dado por la historiografía a la República ${ }^{17}$, sino que los mismos actores coetáneos pensaron el momento que estaban viviendo como una coyuntura experimental. Si nos planteamos, de hecho, la pregunta que Carlo Galli se hacía en un congreso reciente dedicado a los debates filosófico-políticos durante la República de Weimar ${ }^{18}$, "Weimar, ¿laboratorio de qué?", encontramos una respuesta precisa en el texto de Paul Merker:

En virtud de su situación estratégica, de su preponderante emplazamiento industrial en el continente y de su tradición militarista, Alemania fue elegida por todos los enemigos del progreso como el grupo central de los Ejércitos antibolcheviques. Y así debía convertirse, al propio tiempo, en laboratorio para precisar si el fascismo es efectivamente, la forma mejor y más efectiva de ataque y defensa de los grandes monopolios ${ }^{19}$.

Para apoyar su hipótesis, que explica tanto la República de Weimar como el fascismo y el nazismo como meros ensayos del gran capital para frenar el empoderamiento obrero, Merker recurre a una declaración explícita del que fuera hombre más rico del mundo durante las décadas de los veinte y treinta y principal financiador del nazismo y el paramilitarismo antibolchevique, el magnate petrolero holandés Henri Deterding ${ }^{20}$, quien en 1929 afirmaba sobre la democracia de Weimar y el futuro de corte fascista al que él y otros grandes capitalistas la conducían:

El fascismo en Italia no ha facilitado aún la prueba de que es en realidad lo que necesitamos. Sólo en Alemania puede obtenerse esta demostración. Alemania será el campo de experimentación decisivo para saber si el fascismo es en efecto la mejor forma de Gobierno para el mantenimiento y fomento de la fuerza productiva y conservadora. Debe hacerse el ensayo. Mucho se ventila para nosotros en su éxito, si no se ventila todo ${ }^{21}$.

"Prueba", "demostración", "campo de experimentación" y "ensayo" aparecen nítidamente en la familia

17 La primera amplia conceptualización de la República de Weimar como un laboratorio, precisamente, en el campo de los conflictos laborales y el derecho del trabajo la encontramos en E. Fraenkel, G. Arrigo y G. Vardaro (eds.), Laboratorio Weimar: Conflitti e diritto del lavoro nella Germania prenazista, Roma, Edizioni Lavoro, 1982.

18 Congreso Internacional Laboratorio Weimar: La Crisis de la Primera Globalización en Euroamérica (1918-1933), 13-15 de noviembre de 2017, Universidad Complutense de Madrid. Congreso dirigido por José Luis Villacañas.

19 P. Merker, "Prólogo al lector latinoamericano", en P. Merker, La caída de la República Alemana. El camino de Hitler al poder, op cit., p. 16.

20 Principal accionista de la petrolera Shell.

21 Ibidem. conceptual o campo semántico empleado por Deterding. $\mathrm{Su}$ análisis es tan realista como espeluznante, pues revela la naturaleza de la ciencia y filosofía políticas elaboradas durante la República de Weimar como una ciencia de organización y control de las masas.

La misma República y su democracia parlamentaria, en convivencia durante más de una década con la represión paramilitar y por métodos extralegales del movimiento obrero, aparecen como un burdo artificio de contención, necesario tan sólo hasta que pudiera instaurarse un gobierno totalitario que, apoyado por una parte sustancial del pueblo alemán, pudiera dedicarse plenamente a la extirpación del comunismo a escala estatal e internacional.

En la concepción de la política de Deterding no encontramos ciudadanos ni individuos, sino meras "fuerzas" a canalizar y corregir. Queda patente que no sólo Weimar, sino también el fascismo italiano y el totalitarismo nazi, con sus millones de muertos, serían meras pruebas de ensayo y error en el propósito de los grandes capitalistas por imponer su dominio de clase. La influencia directa que Shell -la compañía petrolera que Deterding dirigió- y otras petroleras siguen ejerciendo en el gobierno de muchos países del mundo ${ }^{22}$-en clara violación al principio democrático de la soberanía popular- hacen que no sea descabellado plantear si buena parte de las democracias burguesas y regímenes pseudo-democráticos actuales, cada vez más cercanos al tipo de uso estratégico del terror, los procedimientos de actuación excepcional y represión política que Merker denunciaba como elementos centrales en la República de Weimar, pueden ser consideradas como una nueva fase experimental de esta dominación histórica.

\section{El prólogo exculpatorio "al lector latinoamericano"}

Pese a la relativa libertad de la que gozaban los comunistas alemanes en su exilio mexicano, ésta no era tampoco total. Los condicionantes a la hora de elaborar una historia crítica de la República de Weimar desde México son tenidos muy en cuenta por Paul Merker en el "Prólogo a la edición para América Latina". Tienen que ver, en primer lugar, con la prohibición constitucional ${ }^{23}$ que impide, todavía hoy, a los extranjeros opinar públicamente, manifestarse o incidir activamente en la vida política mexicana. Por ello, exhorta Merker en este "Prólogo" a que no se sacaran implicaciones de su obra para el contexto mexicano, escenario que él no conocía bien ni había tenido en cuenta durante el proceso de redacción de su libro. Merker pide concretamente a sus lectores y al público mexicano, que su escrito no fuera esgrimido contra el país y gobierno que, hospitalaria y fraternalmente, lo había acogido. Su principal preocupación es

\footnotetext{
El prestigioso diario británico The Guardian se hacía eco el 8 de diciembre del 2010 del control directo ejercido por Shell sobre la política energética del gobierno nigeriano, "WikiLeaks cables: Shell"s grip on Nigerian state revealed", https:/www.theguardian.com/business/2010/dec/08/wikileaks-cables-shell-nigeria-spying. Consultado por última vez el 13 de diciembre de 2018.

23 Esta prohibición está recogida en el artículo 33 de la Constitución mexicana vigente desde 1917.
} 
que, dada la marcada hostilidad hacia el SPD y su papel en la deriva imperialista de la República de Weimar que rezuma cada página de La caída de la República Alema$n a$, el libro fuera percibido por los enemigos izquierdistas del gobierno mexicano como una crítica a las líneas marcadamente reformistas y social-demócratas que habían guiado el gobierno de Cárdenas y que, en la época en que se publicó el libro, seguía implementando su sucesor, Manuel Ávila Camacho ${ }^{24}$.

El "Prólogo" representa, sin duda, una contradicción abierta entre las posiciones revolucionarias sostenidas por Merker tanto en su libro como en su condición de militante del KPD durante la República de Weimar y el respaldo a la política socialdemócrata -en defensa de un frente nacional que amalgamara a la burguesía nacional y las corporaciones obreras- que abanderaba el Partido de la Revolución Mexicana (PRM) a comienzos de la década de 1940.

Pese a estas contradicciones y a que, como comunista alemán e internacionalista, sus posiciones estaban muy alejadas del obrerismo populista del PRM, Merker -seguramente influido por el propio Lombardo Toledano- aceptó escribir el "Prólogo" que abre la edición en castellano de su libro.

De forma escueta y sin profundizar mucho en sus argumentos de respaldo al gobierno mexicano -algo que quizás podía comprometerlo en un futuro donde los gobiernos claramente proletarios fueran expandiéndose-, Merker se limitó a salvar las apariencias para contentar, así fuera formalmente, a los gobernantes del país que lo había acogido. En el texto añadido a la edición castellana apunta como argumento legitimador del gobierno mexicano, la existencia de una diferencia sustancial de instituciones y circunstancias históricas en Alemania y América Latina, diversidad de contexto y estructuras que, necesariamente, debía llevar aparejada una diferencia de criterios valorativos en cuanto a las estrategias adoptadas por el proletariado para la defensa de sus intereses y la toma o participación en el poder. En sus propias palabras: "En él (en el libro) se clasifican como reaccionarias circunstancias e instituciones existentes bajo el régimen democrático en Alemania que en los países latinoamericanos precisamente se valoran como progresistas" 25 .

La apelación a esta especificidad nacional o continental mencionada por Merker en su "Prólogo" fue en realidad uno de los principales argumentos de los gobiernos post-revolucionarios para implementar un marxismo muy mitigado en México, distanciándose de los regímenes socialistas de inspiración soviética y posicionándose, desde el final de la Segunda Guerra Mundial, en clara sintonía con el Estados Unidos de Roosevelt y su política de apoyo a la gran industria nacional combinada con el intervencio-

\footnotetext{
24 Precisamente en el sexenio 1940-46, el presidente Ávila Camacho dio un giro hacia el centro para evitar lo que la burguesía crítica con Cárdenas percibía como implantación de un régimen socialista en México. Resulta un elemento difícil de valorar, pero quizás el apoyo ofrecido por Lombardo a la publicación del libro de Merker y a la traducción al castellano de la parte dedicada a Weimar sea una de las pequeñas pullas lanzadas a Ávila y a quienes guiaban a la República Mexicana por un camino cada vez más afín al de la fallida república burguesa de Weimar.

25 P. Merker, "Prólogo al lector latinoamericano", en P. Merker, La caída de la República Alemana. El camino de Hitler al poder, op. cit., p. 19.
}

nismo estatal en sectores estratégicos (electricidad, petróleo, etc.) y una asistencia social con recursos limitados.

Más que por Merker, el "Prólogo a la edición para América Latina" parece escrito por el propio Lombardo, ya que la apelación a la singularidad mexicana para justificar una vía propia al socialismo es el leivmotiv que se repite machaconamente a lo largo de toda su obra ${ }^{26}$. Por su parte, tras algunos años de colaboración con Lombardo en esta lucha por un socialismo a la mexicana, filósofos revolucionarios internacionalistas como José Revueltas se acabaron alejando de la misma y denunciaron "la vía mexicana al socialismo" como la artimaña con la que la burguesía nacional y sus portavoces habían seducido al proletariado mexicano para integrarlo en un proyecto político que nada tenía que ver con sus intereses de clase y postergar sine die la revolución social de la que México estaba urgido.

No podríamos anteponer así, el examen de la realidad concreta de México, al problema de la perspectiva general de la conciencia de la época contemporánea sin que inevitablemente la cuestión se redujera a un litigio local [...], lo que ocurre con la conciencia proletaria en México debe examinarse dentro del conjunto de una situación mundial en la que no hay clase obrera ni pueblo de ningún país que no esté llamado a desempeñar algún papel ${ }^{27}$.

El "Prólogo" al lector mexicano y latinoamericano es, en definitiva, una especie de excusa no pedida en la que Merker intenta dignificar a la República Mexicana que lo acogía como refugiado en ese momento frente a la República de Weimar, tan parecida en algunos aspectos al México de Ávila Camacho y que, sin embargo, Merker había caricaturizado como una mera antesala del nazismo.

\section{Afinidades y diferencias entre la república revolu- cionaria mexicana y la democracia imperialista de Weimar}

Si volvemos de nuevo al contexto mexicano en el que Merker publicó su obra, cabe plantearse en qué clave interna podían ser recibidas sus críticas a Weimar. La perspectiva marxista y radicalmente anti-burguesa de Merker lo lleva a un escepticismo anti-parlamentario y contrario a la vía reformista alentada por el SPD, al que ataca por haber seducido al proletariado alemán con sus "ilusorias teorías de la democracia económica

V. Lombardo Toledano, Las corrientes filosóficas en la vida de México, México, Universidad Obrera de México, 1963, pp. 79-84; V. Lombardo Toledano, ¿Moscú o Pekín?: la vía mexicana hacia el socialismo, México, [Partido Popular Socialista], 1963.

27 J. Revueltas, Un proletariado sin cabeza, en J. Revueltas, Obras completas, Vol. 17, México, Ediciones Era, 1962, p. 51. Y más adelante agrega: "la realización en el poder de la conciencia proletaria en los países dependientes se plantea en la actualidad, durante su primera fase, como la realización nacional del país, esto es, como una erradicación terminante y absoluta del poder imperialista. Ahora bien: esta realización nacional del país, sin embargo, la lleva a cabo (debe llevarla a cabo) la conciencia nacional del proletariado, no la conciencia nacional de la burguesía que en cualquier momento se puede colocar al servicio de los intereses y combinaciones internacionales del imperialismo. La nacionalización de las empresas imperialistas, por ejemplo, no será una nacionalización burguesa, sino una nacionalización proletaria", ibidem, p. 71. 
y la transformación pacífica del capitalismo de acuerdo al interés socialista" ${ }^{28}$.

En el contexto mexicano, tal posición podía ser interpretada bien como un respaldo a un modelo de socialismo revolucionario como el cardenista en el que -al menos en esta época- el Partido de la Revolución Mexicana $^{29}$ fungía como una especie de partido único en el que confluían las organizaciones paraestatales obreras, campesinas, populares y militares. Sin embargo, en la coyuntura por la que atravesaba el régimen en la década de los cuarenta, los ataques de Merker al SPD podían ser leídos también como un ataque a la táctica contemporizadora adoptada por los gobiernos de Cárdenas y Ávila Camacho y a sus guiños conciliadores a los industriales nacionales y la pequeña y mediana burguesía urbana, ante los que se presentaban como una alternativa aceptable a un sistema netamente comunista.

Otro de los puntos que podía generar ficciones radica en el uso constante y sistemático que el Partido de la Revolución Mexicana y sus antecesores habían hecho del nacionalismo desde el triunfo de la Revolución en $1917^{30}$. Las críticas feroces de Merker al reaccionario y excluyente nacionalismo alemán y su perspectiva del pangermanismo y teorías raciales imperialistas como meras fases de un desarrollo lineal de esta matriz nacionalista ${ }^{31}$, podían generar suspicacias y ser vistas en México como una crítica a la ideología oficial del partido gobernante, un nacionalismo antiimperialista con un ligero barniz socialista.

Como sus satíricas andanadas contra la tibieza reformista de los socialdemócratas y el patrioterismo alemán, también los ataques al militarismo prusiano-germano podían ser vistos con recelo en una República Mexicana, que tras la Revolución de 1917, tardó casi tres décadas ${ }^{32}$

28 P. Merker, La caída de la República Alemana. El camino de Hitler al poder, op. cit., p. 147.

29 El presidente Calles había creado el Partido Nacional Revolucionario, activo hasta 1938, en que se refunda como Partido de la Revolución Mexicana. En 1946, durante la presidencia de Ávila Camacho, el PRM fue rebautizado como Partido de la Revolución Institucional (PRI).

30 Comenzando por el mismo logo del partido, que hacía suyos los colores de la bandera mexicana, el nacionalismo resultaba omnipresente en una revolución institucionalizada a apoyar obligatoriamente por todos los verdaderos patriotas.

31 Ideas expuestas en la Segunda Parte de su obra Deutschland-Sein oder Nicht sein? Esta Segunda Parte fue publicada en México en 1945, poco después de la derrota del régimen nazi. Se publicó sólo en alemán con el título Das Dritte Reich und sein Ende. El análisis de las que Merker llama "ideologische Grundlage der nazistischen Politik" figura en las pp. 17-31. Pese al apoyo prestado al intelectual alemán para que publicara su ensayo histórico en la editorial El libro libre, sus tesis tampoco hubieran podido recabar un apoyo completo por parte del mismo Lombardo Toledano. Pese a sus flirteos con el internacionalismo socialista, Lombardo trabajó a lo largo de su trayectoria política y filosófica en una perspectiva netamente mexicanista, de ahí que tras el gobierno de Cárdenas y pese a su sustitución al frente de la gran central sindical mexicana, la Confederación de Trabajadores de México (CTM), por el discutido Fidel Velázquez, decidiera plegarse a la línea adoptaba por Ávila Camacho, al que sólo ofreció una resistencia esporádica y testimonial: Lombardo había apoyado a Ávila durante la coyuntura sucesoria y lo había presentado como el perfecto continuador de la obra cardenista. Se trata de un error de cálculo "que apartó para siempre a la izquierda del gobierno", G. Carcía Cantú, El pensamiento de la Reacción Mexicana. Historia documental. Tomo III (1929-1940), México, UNAM, 1997, p. 249.

32 Con la presidencia de Miguel Alemán Valdés, jurista de formación, a partir de 1946. Aunque hubo otros presidentes civiles entre 1917 en depositar el poder en manos de un presidente civil. En la época en la que escribía Merker, 1944, tal relevo todavía no se había producido, pues al igual que su predecesor Lázaro Cárdenas, Ávila Camacho era un oficial militar de alta graduación y había hecho toda su carrera política dentro de las filas del ejército.

Paul Merker trata de camuflar de alguna manera estos parecidos razonables entre Weimar y México en el "Prólogo al lector latinoamericano" que mencionábamos antes. Merece la pena detenernos en él en la medida en que, en lugar de insistir entre los puntos de afinidad entre Weimar y México, Merker subraya los elementos divergentes en su esfuerzo por presentar al México de la época como una república antagónica a la República de Weimar, nacida muerta. Desde esta perspectiva, si para Merker Weimar representa "el laboratorio de los enemigos del progreso", la República Mexicana es un ensayo y una muestra del progreso resultante allí donde "la revolución democrática conquistó una victoria plena" ${ }^{33}$. Diferenciando a una y otra repúblicas, sostiene Merker en primer lugar:

La estimación diferencial de estas circunstancias e instituciones se desprende de lo siguiente: La democracia de Weimar en Alemania bajo ningún concepto puede equipararse, lo decimos nosotros, con la democracia que en México prevalece. Incluso bajo la democracia de Weimar, Alemania continuó siendo un país imperialista en el que dominaba la clase de monopolistas y Junkers más agresiva del mundo. Por el contrario impera en México una democracia ajena a todos los afanes expansionistas del imperialismo ${ }^{34}$.

En efecto, pese a la derrota de 1918, el pangermanismo siguió ocupando un lugar central en el ideario político alemán durante la República de Weimar, hasta el punto de que la "aspiración de Alemania a tener colonias" 35 quedó recogida en los artículos 6 y 80 de la

y 1946, se trata de presidentes interinos que gobernaron en períodos cortos y bajo el control de generales como Plutarco Elías Calles.

33 P. Merker, "Prólogo al lector latinoamericano", en P. Merker, La caída de la República Alemana. El camino de Hitler al poder, op. cit., p. 19.

34 Ibidem.

35 Ibidem. La posibilidad de que otros "países de la región alemana" se incorporaran al Reich quedaba también abierta en el significativo artículo 2 , incentivador del derecho de las poblaciones de estos países a su autodeterminación como ciudadanos de la República alemana. Merker lanza también una mirada crítica a la vida cultural de Weimar. La producción literaria de la República es percibida desde un punto de vista marxista como mera superestructura ideológica al servicio de las clases dominantes, esos "monopolistas, nobles, altos militares y grandes burgueses" cuya supervivencia y futuro como clase social dependía del acceso a un mercado colonial de recursos naturales poco costoso, a conseguir por la fuerza, P. Merker, Deutschland-Sein oder Nicht sein? Band 2. Das Dritte Reich und sein Ende, op. cit., p. 28. Para servir tales objetivos y el interés de los grupos mencionados, las autoridades de Weimar permitieron conservar sus cátedras de filosofía, antropología, biología, historia o derecho a los reaccionarios pangermanistas que habían alentado la Primera Guerra Mundial, mientras que, gracias a la inacción o la complicidad de las fuerzas de seguridad, "científicos progresistas" como los filósofos Theodor Lessing (fue destituido por el rector de la Universidad de Hannover en 1926 tras escribir un artículo crítico contra Hindenburg y sufrir las iras de los estudiantes conservadores) y Friedrich Wilhelm Foerster (en medio de amenazas de muerte y atentados abandonó la Universidad de Münich ya en 1922) o el matemático y escritor político Emil Julius Gumbel (destituido de su 
Constitución. Como bien hacía constar Merker, tal situación no se daba, de ninguna manera, en México, donde la ideología en boga era, ciertamente, un patriotismo, pero de corte anticolonial y antiimperialista, siendo la máxima aspiración del gobierno revolucionario el lograr la independencia real de México frente a todas a las potencias imperialistas de Europa y Estados Unidos que habían atormentado a México durante su primer siglo de existencia independiente y cercenado su territorio.

Quizás sea precisamente Lombardo Toledano el mejor exponente de este nacionalismo anti-imperialista en el México revolucionario. Es, en todo caso, el elemento que vertebra su amplísima obra ${ }^{36}$, exposición pionera de los principales elementos comunes a los populismos latinoamericanos. También en la "Introducción" que escribió a la obra de Merker sobre Weimar dejó algunas pinceladas de una ideología anti-colonial destinada a transformar el mundo en las décadas siguientes. Dando por hecho "la victoria de las Naciones Unidas" en la guerra contra Hitler, Lombardo Toledano prevé, de hecho, los procesos de descolonización y los llama, sintomáticamente, "la segunda fase de la guerra contra el fascismo". En su perspectiva, por tanto, la derrota de la Alemania nazi y su visión del mundo como un espacio a conquistar y dominar por las razas superiores, resulta tan sólo una primera fase en la gran batalla contra el imperialismo que se avecinaba en África, Asia y América y de cuyo resultado dependía el logro de "una paz justa y duradera". Afirma Lombardo en este sentido:

Pero la paz será justa y duradera sólo en el caso de que lleve a todos los pueblos, grandes y chicos, el derecho a elegir su propia forma de vida, el derecho a decidir su propio futuro, a administrar sus asuntos políticos, económicos y culturales, como lo juzguen más conveniente, en tanto que no se mezclen en los asuntos de los demás ni permitan el retorno del fascismo ${ }^{37}$.

Al margen del diferente carácter de los nacionalismos mexicano y alemán, Merker entra en este "Prólogo al lector latinoamericano" en muchos otros elementos

cátedra en la Universidad de Heidelberg en 1932 por el entonces Ministro del Culto en el Estado de Baden, Eugen Baumgartner, del Zentrumspartei) tuvieron que abandonar sus cátedras ya en la década de los veinte, ante la violenta persecución que sufrían a diario por parte de jóvenes nacionalsocialistas y conservadores. Habla Merker de una auténtica entrega de la esfera de la opinión pública a defensores del racismo y el imperialismo como Oswald Spengler (autor del famoso ensayo racista La decadencia de Occidente, publicado entre 1918 y 1923) como una "mancha que los gobiernos de la República y sus partidos nunca podrán limpiarse". Su juicio cobra gran actualidad hoy en día, cuando partidos racistas, antisemitas o islamófobos vuelven a ser normalizados y aceptados como actores del juego parlamentario burgués.

36 Acle-Kreysing ha subrayado recientemente, en términos generales, la importancia en el México postrevolucionario de este nacionalismo orientado originalmente en clave antifascista y, posteriormente, en perspectiva antiimperialista, "Antifascismo: un espacio de encuentro entre el exilio y la política nacional. El caso de Vicente Lombardo Toledano en México (1936-1945)", op. cit. La autora alemana llega a considerar al "nacionalismo revolucionario" como "una especie de ideología oficial en México" hasta la década de los ochenta, ibidem, p. 578 .

37 V. Lombardo Toledano, "Introducción", en P. Merker, La caída de la República Alemana. El camino de Hitler al poder, op. cit., p. 13. que, en su opinión, distinguían a las repúblicas de Weimar y México. Sin espacio para entrar en muchos detalles sobre esta amplia comparación, mencionemos tan sólo que allí donde Merker veía a un Weimar tutelado por militares belicosos y revanchistas, encuentra un México militarizado para protegerse ante los ataques imperialistas de Estados Unidos, Gran Bretaña o Francia, las potencias coloniales que habían llevado a México a perder más de la mitad de su territorio en el siglo XIX.

Otro punto de comparación tiene que ver con el rol de los sindicatos, leyes sobre el salario y órganos de arbitraje entre patrones y obreros. Mientras que los creados por la Alemania de Weimar le parecen una mera coartada para prohibir las huelgas y reprimir el auténtico movimiento obrero, los instaurados por los gobiernos mexicanos tras la Revolución le parecen valiosos instrumentos de apoyo del gobierno a obreros y sindicatos en la defensa de sus reivindicaciones frente a los "patrones económicamente poderosos"38. Otros aspectos que elogia en la República Mexicana frente a Weimar son la Reforma Agraria -en la medida en que su núcleo esencial consistió en la disolución de los grandes latifundios privados y el apoyo a la creación de ejidos o tierras comunales- y la amplia legislación social aprobada entre 1917 y 1944.

\section{Conclusiones. Lombardo Toledano y el (neo)nazismo, una relación duradera}

En este artículo nos hemos concentrado en la obra de Merker La caída de la República Alemana, uno de los veintiséis libros de esa interesante iniciativa editorial germano-mexicana, de clara orientación antifascista, que fue El libro libre. Sería interesante poder seguir las huellas que el pensamiento político alemán de los cuarenta dejó en México y rastrear este influjo en una perspectiva más amplia. Se observa, por ejemplo, de modo nítido en el caso de la obra de Lombardo Toledano, influido por las perspectivas de Merker incluso muy posteriormente. Por ejemplo, en un libro de Lombardo llamado El Neonazismo, sus caracteristicas y sus peligros, resultado de dos conferencias impartidas en la Universidad Nacional Autónoma de México (UNAM) en 1960, Lombardo realizó él mismo una reconstrucción histórica de las fuerzas políticas y económicas que llevaron a Hitler al poder y de los distintos factores que provocaron la caída de la República de Weimar. Pese a que la Segunda Guerra Mundial había concluido ya tres lustros antes, Lombardo seguía considerando vivas las motivaciones e inquietudes de Merker y enfatizaba la precaución que debían tener la República Mexicana y el mundo libre ante un fascismo aún vivo, en la medida en que la pervivencia de este pensamiento y las innegables continuidades estructurales existentes entre el Tercer Reich y la Europa de la OTAN y las Comunidades Europeas, todavía inmersa en multitud de guerras coloniales, hacía cada vez mayor el riesgo de una nueva guerra mundial.

\footnotetext{
38 P. Merker, "Prólogo al lector latinoamericano", en P. Merker, La caída de la República Alemana. El camino de Hitler al poder, op. cit., p. 20.
} 
Entre otras cosas, Lombardo arroja la sospecha sobre el rápido restablecimiento de relaciones pacíficas entre la Alemania Federal y sus supuestos enemigos occidentales (Francia, Gran Bretaña, Estados Unidos), quienes después de las dos guerras mundiales contribuyeron a la división de Alemania, al rearme de la Alemania Occidental como vanguardia antisoviética -en un ejército plagado de antiguos altos oficiales nazis, cuyo recuento proporciona Lombardo de forma exhaustiva en esta obra- y a la reconstrucción de la misma Alemania Federal mediante el Plan Marshall y una amplia serie de créditos y negocios en común. Como observa Lombardo, buena parte de los grandes grupos o clanes empresariales que, disputándose la hegemonía en Alemania para convertirse en monopolios, habían apoyado a partidos cada vez más reaccionarios y financiado y llevado al poder a Hitler, seguían dirigiendo la economía de Alemania. En la medida en que siguen conduciendo "la locomotora de Europa" hasta nuestros días, el análisis histórico-filosófico y político realizado por Lombardo en los sesenta sigue teniendo actualidad.

Siguiendo la idea rectora expuesta por Merker, para Lombardo, Weimar, el nazismo, la Segunda Guerra Mundial y las alianzas antisoviéticas durante la Guerra Fría no son más que episodios de una misma estrategia o "laboratorio" de la burguesía internacional contra el comunismo, cuya consigna siempre fue muy clara: "La marcha hacia el este del imperialismo alemán tiene una misión más alta, la destrucción del primer régimen socialista de la historia, por eso hay que perdonarlo y ayudarlo a que recupere las fuerzas perdidas y las mejore" 39 .

Este trabajo tardío de Lombardo resulta especialmente interesante por su uso pionero del término "neonazismo", empleado ya por el filósofo mexicano a comienzos de la década de los sesenta. De igual modo, la concepción de Weimar como laboratorio y experimento cobran en el contexto político actual, en el que las derechas europeas ya no se presentan unidas en partidos articulados por el liberalismo y un conservadurismo moderado, sino fragmentadas en diversas agrupaciones cuyo radicalismo xenófobo y ultraderechista va creciendo de año en año y elección en elección, una gran actualidad. En este sentido, sigue vigente la idea expuesta por Lombardo Toledano de que "el fascismo es la dictadura del capital financiero y constituye la filosofía social del imperialismo"40 y de que, mientras que no se implementen políticas destinadas a dificultar la conformación de este tipo de capitales y su expansión global, favoreciendo una economía productiva regulada y dirigida democráticamente por las clases trabajadoras, las lacras del fascismo y el imperialismo no sólo no se erradicaran, sino que continuarán perviviendo e imperando en tanto que reflejo necesario de unas relaciones de producción pervertidas.

Como Lombardo subraya en la parte final de $E l$ Neonazismo, sus caracteristicas y sus peligros, la prohibición de los carteles y monopolios en los tratados de Postdam (1945) fue una especie de reconocimiento implícito y a posteriori por parte de las potencias capitalistas de las verdaderas causas de la guerra y de la complicidad y continuidad existente entre los partidos políticos centristas y conservadores en la República de Weimar y el Tercer Reich. Sin centrarse en el "monstruo" Hitler, tanto Lombardo como Merker ponen el foco en los verdaderos actores colectivos detrás de los individuos-instrumento que se vieron implicados en la Segunda Guerra Mundial, resultado de un desastroso experimento político, de una larga serie de ensayos de laboratorio largo tiempo planeados con el fascismo italiano como antecedente inmediato- para frenar el incipiente movimiento bolchevique. Aunque, para regocijo de sus impulsores, el experimento logró sepultar durante varios años los ideales democráticos y obreristas en Europa, acabó derrotado en una nueva guerra mundial que no sólo asestó un varapalo a las ambiciones de hegemonía global de los grandes consorcios europeos, sino que dio lugar a que el comunismo socializador de los medios de producción se extendiera hasta la misma Alemania y por todos aquellos países de la Europa central y oriental que el nazismo había considerado como su campo natural de expansión.

Postdam fue, por tanto, el reconocimiento de que Merker y los comunistas alemanes no habían estado equivocados al subrayar la complicidad de la burguesía en la deriva política de Weimar y de las élites financieras en la progresiva consolidación de una economía de guerra. Desde esta perspectiva, Hitler y su astuto uso del discurso del odio y el revanchismo se presentan sólo como una evolución ulterior de una serie de dinámicas desatadas por el pacto entre el socialdemócrata Ebert y el militarismo prusiano de finales de la década de 1910. Nada más actual, por tanto, que las palabras con las que Lombardo definía el neonazismo en 1960 $\mathrm{y}$, en general, las estrategias de dominación de carácter burgués-fascista: "El fascismo se puede definir como la dictadura terrorista de los elementos más reaccionarios del capitalismo financiero" 41 .

\footnotetext{
39 V. Lombardo Toledano, El neonazismo: sus características y sus peligros, México, Escuela Nacional de Ciencias Políticas y Sociales, 1960 , p. 30.

$40 \quad$ Ibidem, p. 47.
} 


\section{Bibliografía}

Abellán, J. L., “Manuel Andújar, literatura y conciencia”, Cuadernos Hispanoamericanos 529-530, 1994, pp. $281-292$.

Acle-Kreysing, A., "Antifascismo: un espacio de encuentro entre el exilio y la política nacional. El caso de Vicente Lombardo Toledano en México (1936-1945)”, Revista de Indias LXXVI, 267, 2016, pp. 573-609.

Cañadas García, T., La huella de la cultura en lengua alemana en México a partir del exilio de 1939-1945, Madrid, Fundación Universitaria Española, 2016.

Cózar, R. De, "Manuel Andújar y el exilio", Boletín del Instituto de Estudios Giennenses 211, 2015, pp. 281-292.

Flechtheim, O., Der KPD in der Weimarer Republik, Offenbach, Bollwerk/Karl Drott, 1948.

Fraenkel, E. /Arrigo, G./Vardaro, G. (eds.), Laboratorio Weimar: Conflitti e diritto del lavoro nella Germania prenazista, Roma, Edizioni Lavoro, 1982.

García Cantú, G., El pensamiento de la Reacción Mexicana. Historia documental. Tomo III (1929-1940), México, UNAM, 1997.

Hanffstengl, R., Mexiko, das wohltemperierte Exil, México, Instituto de Investigaciones Interculturales Germano-Mexicanas, 1995.

Hervás Fernández, G., "Manuel Andújar, un escritor del exilio", Revista de Filología Románica 28, 2011, pp. 163-170.

Janzen, M., Writing to Change the World: Anna Seghers, Authorship, and International Solidarity in the Twentieth Century, Rochester, Candem House, 2018.

Katz, F., "El exilio centroeuropeo. Una mirada autobiográfica”, en Yankelevich, P., México, país refugio: la experiencia de los exilios en el siglo XX, México, CONACULTA / INAH, 2002, pp. 43-48.

Kießling, W., Paul Merker in den Fängen der Sicherheitsorgane Stalins und Ulbrichts, Berlín, Gesellschaftswissenschaftliches Forum, 1995.

-, Partner im Narrenparadies. Der Freundeskreis um Noel Field und Paul Merker, Dietz Verlag, Berlin, 1994.

-, Viva Mexico. Brücken nach Mexiko. Tradition einer Freundschaft, Dietz Verlag, Berlin, 1989.

-, Exil in Lateinamerika. Kunst und Literatur im antifaschistischen Exil 1933-1944, Leipzig, Reclam, 1980.

-, Alemania Libre in Mexiko. Band 1. Ein Beitrag zur Geschichte des Antifaschistischen Exils 1941 - 1946, Berlín, Akademie Verlag, 1974.

-, Alemania Libre in Mexiko. Band 2. Texte Und Dokumente Zur Geschichte Des Antifaschistischen Exils 1941- 1946, Berlín, Akademie Verlag, 1974.

Kinner, K., Der deutsche Kommunismus 1. Die Weimarer Zeit: Selbstverständnis und Realität, Berlin, Dietz, 1999.

Lombardo Toledano, V., Las corrientes filosóficas en la vida de México, México, Universidad Obrera de México, 1963.

-, El neonazismo: sus características y sus peligros, México, Escuela Nacional de Ciencias Políticas y Sociales, 1960.

Merker, P., Deutschland - Sein oder Nicht Sein? Band 2. Das Dritte Reich und sein Ende, México, El Libro Libre, 1945.

-, La caída de la República Alemana. El camino de Hitler al poder, México, El Libro Libre, 1944.

-, Deutschland - Sein oder Nicht Sein? Band 1. Von Weimar zu Hitler, México, El Libro Libre, 1944.

Pérez Montfort, R., "Apuntes sobre el exilio alemán en México", en Gleizer, D., Unwelcome exiles: Mexico and the Jewish refugees from Nazism, 1933-1945, Leiden, Brill, 2013, pp. 49-54.

Pohle, F., Das Mexicanische Exil: ein Beitrag zur Geschichte der politisch-kulturellen Emigration aus Deutschland (1937-1946), Stuttgart, Metzler, 1986.

Vegazo Palacios, V., "Manuel Andújar, el exilio y la vindicación de la memoria histórica", Campo de Agramante: Revista de literatura 9, 2008, pp. 95-108.

Wirsching, A., Vom Weltkrieg zum Bürgerkrieg? Politischer Extremismus in Deutschland und Frankreich 1918 - 1933/9, München, Oldenbourg, 1999. 